\title{
Irisin ameliorates nicotine-mediated atherosclerosis via inhibition of the PI3K pathway
}

\author{
Kang $\mathrm{Li}^{1}{ }^{\wedge}$, Junye Chen ${ }^{1} \wedge$, Chaonan Wang ${ }^{1}$, Jiang Shao ${ }^{1}$, Zhichao Lai ${ }^{1}$, Xiaoxi Yu ${ }^{1,2}$, Fenghe Du ${ }^{1,3}$, \\ Ran $\mathrm{Gao}^{4}$, Jing Wang ${ }^{4}$, Bao $\mathrm{Liu}^{1} \wedge$
}

${ }^{1}$ Department of Vascular Surgery, Peking Union Medical College Hospital, Chinese Academy of Medical Science, Beijing, China; ${ }^{2}$ Eight-year Program of Clinical Medicine, Peking Union Medical College Hospital, Peking Union Medical College, Chinese Academy of Medical Sciences, Beijing, China; ${ }^{3}$ Four-Year Program of Clinical Medicine, Peking Union Medical College Hospital, Peking Union Medical College, Chinese Academy of Medical Sciences, Beijing, China; ${ }^{4}$ State Key Laboratory of Medical Molecular Biology, Institute of Basic Medical Sciences, Chinese Academy of Medical Sciences, Department of Pathophysiology, Peking Union Medical College, Beijing, China

Contributions: (I) Conception and design: B Liu, J Wang, K Li; (II) Administrative support: B Liu, J Wang; (III) Provision of study materials or patients: K Li, J Chen; (IV) Collection and assembly of data: K Li, J Chen, C Wang; (V) Data analysis and interpretation: K Li, J Chen, R Gao; (VI) Manuscript writing: All authors; (VII) Final approval of manuscript: All authors.

Correspondence to: Bao Liu. Department of Vascular Surgery, Peking Union Medical College Hospital, Shuaifuyuan 1st, Dongcheng District, Beijing 100730, China. Email: liubao72@aliyun.com.

Background: Atherosclerosis is a chronic disease, with smoking being an independent risk factor. Irisin, a factor produced by myocytes, is expected to treat smoking-related arteriosclerosis, however its specific mechanism remains unclear.

Methods: Forty Apoe-/- mice with nicotine intervention were involved in this study. The atherosclerotic lesions, smooth muscle cell proliferation, and macrophage infiltration induced by nicotine, and the corresponding changes caused by the administration of irisin, were obtained. The integrin $\alpha \mathrm{V} \beta 5$ inhibitor, cilengitide, was included to determine the cell entry pathway of irisin. Proteins and mRNA levels of phosphatidylinositol 3-kinase (PI3K) and downstreams were detected to clarify the specific molecular mechanism of irisin activity.

Results: H\&E staining and Masson staining showed that nicotine could aggravate the intensity of atherosclerosis in mice, and Irisin could reverse the thickening of the vascular media induced by nicotine. Immunohistochemical staining of CD68 and $\alpha$-SMA suggested that Irisin could inhibit nicotine-mediated macrophage infiltration and smooth muscle cell proliferation. The protective effect of Irisin was partially reduced after the administration of cilengitide, confirming that Irisin enters cells through multiple ways, including integrin $\alpha v \beta 5$. Nicotine was confirmed to activate the PI3K pathway to promote media thickening, while Irisin can inhibit the activation of the PI3K pathway, thus playing its anti-atherosclerosis role. Irisin was further observed to reverse nicotine-mediated P27 down-regulation.

Conclusions: Irisin was found to inhibit nicotine-mediated medium thickening, smooth muscle cell proliferation, macrophage infiltration, and atherosclerosis progression via the integrin $\alpha$ V $\beta 5 / \mathrm{PI} 3 \mathrm{~K} / \mathrm{P} 27$ pathway.

Keywords: Irisin; nicotine; atherosclerosis; PI3K; integrin $\alpha \mathrm{V} \beta 5$

Submitted Apr 02, 2021. Accepted for publication May 06, 2021.

doi: $10.21037 /$ atm-21-2072

View this article at: http://dx.doi.org/10.21037/atm-21-2072

\footnotetext{
^ ORCID: Kang Li, 0000-0002-5739-3082; Junye Chen, 0000-0001-9224-1677; Bao Liu, 0000-0001-6359-959X.
} 


\section{Introduction}

Atherosclerosis is a persistent and chronic vascular disease, which could lead to cardio-cerebrovascular diseases, which are the leading causes of disability and mortality worldwide (1). In 2015, approximately 422.7 million people were affected by cardio-cerebrovascular diseases globally, resulting in 17.9 million deaths (which accounts for $31 \%$ of global deaths) (2). Atherosclerosis involves a complex pathological mechanism characterized by the dysregulation of vessel structures. This process involves local inflammation, endothelial disorder, vascular smooth muscle cell proliferation, and angiostenosis.

Smoking has been identified as an independent risk factor for atherosclerotic disease. Nicotine in tobacco is an important component involved in vascular diseases; it binds to nicotinic acetylcholine receptors (nAChRs), which causes conformational changes in these receptors. This in turn regulates $\mathrm{Na}^{+}, \mathrm{K}^{+}$, and $\mathrm{Ca}^{2+}$ ion flow to activate inflammatory pathways and promote the secretion of inflammatory factors, ultimately leading to atherosclerosis (3). Nicotine can damage the normal function of the vascular endothelium and lead to excessive production of endogenous reactive oxygen species (ROS) in vitro (4). In addition, nicotine can also increase the apoptosis and morphological abnormality of vascular endothelial cells, or directly act on the nicotine receptors of smooth muscle cells, thereby inducing the proliferation and migration of smooth muscle cells (5).

In 2012, Boström et al. discovered a new molecule secreted by myocytes, termed irisin, which is a membrane protein fragment of fibronectin type III domain protein 5 (FNDC5) (6) and appears in the form of a homodimer, forming a $\beta$ sheet in between (7). Irisin was found to have the ability to decrease the number of early and late apoptotic macrophages stimulated by lipopolysaccharides and suppress the mitogen-activated protein kinase (MAPK) pathway, which causes decreased phosphorylation of Nuclear Factor

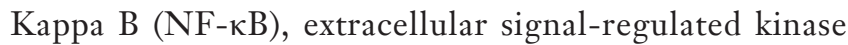
(ERK), and c-Jun N-terminal kinases (JNK). This leads to reduced production of pro-inflammatory cytokines, such as Interleukin- $1 \beta$ (IL-1 $\beta$ ), IL-6, tumor necrosis factor- $\alpha$ (TNF- $\alpha$ ) and Monocyte chemoattractant protein-1 (MCP-1) (8), confirming the protective role of irisin in the development of chronic inflammation-related diseases.

Irisin has also been found to bind to integrin $\alpha 1 \beta 1$ and $\alpha \mathrm{V} \beta 5$ in adipocytes and osteocytes (9). Integrin $\alpha v \beta 5$ inhibitory peptides with Arg-Gly-Asp (RGD) motif could abolish the signal response and function induced by irisin (9). Irisin has been widely used in cardiovascular disease treatment research in recent years. It has been found to have a protective effect on endothelial cells and could reduce blood pressure in spontaneously hypertensive rat models via the adenosine 5'-monophosphate activated protein kinase $(\mathrm{AMPK}) /$ protein kinase B (AKT)/ endothelial nitric oxide synthase (eNOS)/nitric oxide (NO) pathway (10). Li et al. reported that the activation of farnesoid X receptor (FXR) could induce FNDC5 mRNA expression in human hepatocytes and increased the circulating level in Rhesus macaques. Meanwhile, the administration of Irisin on Apoe-/- mice showed improved hyperlipidemia and alleviated atherosclerosis as compared with Apoe-/- mice (11). Accordingly, FNDC5/Irisin is considered as a direct transcriptional target of FXR, and may be a novel therapeutic strategy for dyslipidemia and atherosclerosis. Several studies have mentioned that irisin inhibits neointima formation, endothelial injury and inflammation in Apoe-/- mice model (12), Irisin reduced oxidized low density lipoprotein (oxLDL)-mediated endothelial cell apoptosis, inflammatory cytokines and intracellular ROS levels, and up-regulated p-AKT/ mechanistic target of rapamycin (mTOR)/NF-E2related factor 2 (Nrf2). In addition, mTOR/Nrf2 shRNA and LY294002 both ameliorate the protective effect of irisin, suggesting that irisin alleviates oxLDL-induced vascular injury through activation of Akt/mTOR/Nrf2 pathway (13). Irisin was also included in other chronic disease researches, such as diabetes and obesity. It was reported to play a beneficial role by inducing white adipose tissue browning through p38 and ERK signaling pathway, which is related to weight loss and improvement of glucose homeostasis (14). Irisin could also act on adipose tissue, muscle, liver, and pancreas, and play roles in glucose homeostasis through synergistic action with other hormones (15). FNDC5/Irisin plays a key role in reducing adipose tissue inflammation and M1 type macrophage polarization through AMPK (16), while FNDC5 deficiency reduced insulin sensitivity in obese mice (17). These demonstrated that Irisin is associated with major chronic diseases and may act as a potential therapy.

In this study, we constructed nicotine-intervened Apoe-/mice model to explore the protective effect of irisin on nicotine-mediated atherosclerosis and its molecular mechanism.

We present the following article in accordance with the 
ARRIVE reporting checklist (available at http://dx.doi. org/10.21037/atm-21-2072).

\section{Methods}

\section{Experimental animals}

All animal experiments were conducted according to the guidelines of the National Health and Family Planning Commission of the People's Republic of China and approved by the Animal Ethics Committee of Peking Union Medical College Hospital, Chinese Academy of Medical Sciences (approval number JS-2335).

In order to elucidate the molecular mechanism of irisin ameliorating nicotine-induced atherosclerosis, 40 6-weekold male Apoe-/- C57BL/6J mice (The Charles River Laboratories, USA) were randomly divided into five groups: (I) Control group: $\mathrm{n}=8$; (II) Nicotine group: nicotine (No. 36733, Sigma Aldrich, China) was administrated in drinking water with $100 \mu \mathrm{g} / \mathrm{mL}$ for 8 weeks, $\mathrm{n}=8$; (III) Irisin group: irisin (No. 11451, Cayman, USA) administrations were applied at $2 \mu \mathrm{g}$ in $100 \mu \mathrm{L}$ normal saline per mouse, intravenously (i.v.), and were conducted twice a week from week 5, n=8; (IV) Nicotine + Irisin group: nicotine and irisin were applied as mentioned above, $\mathrm{n}=8$; and (5) Nicotine + Irisin + Cilengitide group: $4 \mu \mathrm{g} / \mathrm{g}$ cilengitide (HY-16141, MedChemExpress, China) in $100 \mu \mathrm{L}$ normal saline, intraperitoneally (i.p.), was approved every other day from week 5 , in addition to nicotine and irisin, $\mathrm{n}=8$. All of the Apoe-/- mice were fed in a standard SPF chamber at room temperature of $20-26^{\circ} \mathrm{C}, 5 \mathrm{mice} / \mathrm{cage}$, humidity range of $40-70 \%, 12 \mathrm{~h} \mathrm{light/dark} \mathrm{cycle,} \mathrm{free} \mathrm{access} \mathrm{to} \mathrm{food} \mathrm{and}$ water, normal diet for 6 weeks and received a high-fat diet from the start of administration for atherosclerosis animal model building. The groups without nicotine intervention were given saline in the same manner. The weight of all mice was measured weekly after the start of the experiment.

\section{Atherosclerotic lesion assay}

Eight weeks after the initiation of the study, the mice were euthanized for further analysis. Normal saline was then used to perfuse through the left ventricle in situ. Excess adipose tissue around the aorta was removed from the root of the aorta to the bilateral iliac arteries. The root of the aorta and the initial segment of the abdominal aorta were separated and fixed overnight with $4 \%$ paraformaldehyde. Fixed arterial roots were cut into $4-\mu \mathrm{m}$-thick sections. Masson's trichrome staining kit (No. G1006, Servicebio, China) and hematoxylin and eosin (HE) staining were used to evaluate the size of atherosclerotic lesions, as well as the content of collagen and muscle fibers in the lesions. Medium thickness was evaluated by CaseViewer (Ver. 2.4, 3DHISTECH, Hungary).

\section{Immunobistochemical staining}

Immunohistochemical staining of the initial segment of the abdominal aorta was conducted to detect the expression levels of $\alpha$-smooth muscle actin ( $\alpha$-SMA, 1:300, ab5694, Abcam, China) and macrophage (CD68, 1:3,000, ab955, Abcam, China). The cross-sectional atherosclerotic area and the positive staining area were quantified by ImageProPlus (Ver. 6.0, Media Cybernetics, USA).

\section{Enzyme-linked immunosorbent assay (ELISA)}

The plasma of the mice was obtained before the end of the experiment. The plasma concentrations of nicotine metabolites and cotinine (zc-38437, ZCIBIO Technology, China) were determined by ELISA kits according to the manufacturer's instructions.

\section{Western blot}

Aortic proteins were lysed and extracted using RIPA buffer (89900, Thermo Scientific, USA) containing a protease and phosphatase inhibitor cocktail (78443, Thermo Scientific, USA). Proteins were separated by SDS-PAGE and transferred to PVDF membranes. The primary antibodies used for immunoblotting were PI3K (1:10,000, YM3408, Immunoway, China), AKT (1:4,000, 4691, CST, USA), p-AKT-T308 (1:1,000, 13038, CST, USA), p-AKT-S473 (1:1,000, 4060, CST, USA), P21 (1:1,000, YM3453, Immunoway, China), P27 (1:1,000, 3686, CST, USA), and $\beta$-Actin (1:5,000, YM3028, Immunoway, China). The immunoreactive bands were displayed using an electrochemiluminescence (ECL) kit (DW101-01, TransGen Biotech, China) and analyzed by ImageJ software (ver. 1.44, National Institutes of Health, USA).

\section{Quantitative RT-PCR}

TRIzol total RNA extraction reagent (15596-018, Invitrogen, USA) was used for sample RNA extraction. RevertAid First Strand cDNA Synthesis Kit (K1622, 
Table 1 Body weights of mice at baseline and at the end of the experiment

\begin{tabular}{lcc}
\hline \multirow{2}{*}{ Group } & \multicolumn{2}{c}{ Body weight $(\mathrm{g})$} \\
\cline { 2 - 3 } & Week 0 & Week 8 \\
\hline Control & $23.6 \pm 1.9$ & $30.2 \pm 4.1$ \\
Nicotine & $23.8 \pm 1.5$ & $30.8 \pm 7.1$ \\
Irisin & $22.4 \pm 1.6$ & $30.1 \pm 5.2$ \\
Nicotine + Irisin & $23.8 \pm 1.6$ & $30.5 \pm 4.6$ \\
Nicotine + Irisin + Cilengitide & $23.9 \pm 1.0$ & $31.3 \pm 5.1$ \\
\hline
\end{tabular}

Note: $\mathrm{n}=8$.

Thermo, USA) and PrimeScript ${ }^{\mathrm{TM}}$ RT Master Mix (RR036A, TaKaRa, Japan) were used for cDNA synthesis and real-time amplification. All experimental operations were performed according to the manufacturer's instructions.

Primer sequence for target genes were as follow: PTEN-F: 5'-ATCCTTCCAAGCAAAGCATC-3', PTEN-R: 5'-CTGAGACATCAAGCCCGTTC -3'; AKT3-F: 5'-AAGGCAGAAGTAGAACCGAG-3', AKT3-R: 5' -ATGGAGACAAGAGGTCAAGG-3'; CDKN1A-F: 5'-AAGCAGTCACAGCCTAGAAC-3', CDKN1A-R: 5'-AGATCACCAGATTAACCCTC -3'; CDKN1B-F: 5'-AAATGTTTCAGACGGTTCCC-3', CDKN1B-R: 5'-ATGCTTTTAGAGGCAGATGG-3'; ACTB-F: 5'-CGTTGACATCCGTAAAGACCTC-3', and ACTB-R: 5'-ACAGAGTACTTGCGCTCAGGAG-3' . All data was applied with $2^{-\Delta \Delta C T}$ method to analyze and normalized to the expression level of ACTB. All values were presented as fold changes to the control group.

\section{Statistical analysis}

GraphPad Prism (ver. 7.0, GraphPad, USA) was used to analyze the data. All results were presented as the mean \pm Standard Deviation (SD). Comparisons between two groups were analyzed using the two-tailed Student's $t$-test, and comparisons between three or more groups were analyzed using one-way ANOVA. Statistical significance was defined as ${ }^{*} \mathrm{P}<0.05,{ }^{* *} \mathrm{P}<0.01,{ }^{* * *} \mathrm{P}<0.001$, and ${ }^{* * *} \mathrm{P}<0.0001$.

\section{Results}

\section{Nicotine increases atherosclerotic lesion size and content}

Forty Apoe-/- mice were randomly divided into five groups, including a Control group, Nicotine group, Irisin group, Nicotine + Irisin group, and Nicotine + Irisin + Cilengitide group. No significant changes in body weight were observed among the five groups (Table 1). The 14-weekold Apoe-/- mice fed with a high-fat diet and drinking water with nicotine showed increased atherosclerosis and medium thickness of the aortic root and ascending aorta (mean 43.06 vs. $68.51 \mu \mathrm{m} ; \mathrm{P}<0.0001$, Figure $1 A, B$ ) compared with 14-week-old Apoe-/- mice fed with a highfat diet and drinking water without nicotine. Compared with the Control group, nicotine intervention also increased macrophage infiltration (CD68 positive area $1.159 \%$ vs. $2.997 \% ; \mathrm{P}=0.0005$, Figure $1 A, C)$ and the number of smooth muscle cells ( $\alpha$-SMA positive area $6.045 \%$ vs. $3.497 \%$; $\mathrm{P}=0.0035$, Figure $1 A, D)$ in the aortic root and ascending aorta of the mice. The nicotine intervention model was successfully constructed, which was consistent with the level of plasma cotinine (Figure 1E).

\section{Irisin could reverse nicotine-induced atherosclerosis}

After 8-weeks of nicotine exposure and 4-weeks of irisin exposure, the Nicotine + Irisin group was observed (using Masson's staining and HE staining) to reverse nicotineinduced atherosclerosis and reduce nicotine-mediated medium thickening (mean 48.49 vs. $68.51 \mu \mathrm{m} ; \mathrm{P}<0.0001$, Figure $1 A, B$ ) in the aortic root and ascending aorta. Irisin was also observed to ameliorate the nicotine-induced infiltration of macrophages (CD68 positive area $1.633 \%$ vs. $2.997 \% ; \mathrm{P}=0.0108$, Figure $1 A, C)$ and the increasing number of smooth muscle cells $(\alpha-S M A$ positive area $4.182 \%$ vs. $6.045 \% ; \mathrm{P}=0.0464$, Figure $1 A, D)$. Among all of the stains, we observed no significant differences in medium thickness, macrophage infiltration, and smooth muscle cell proliferation between the Control, Irisin, and Nicotine + Irisin groups (Figure 1A,B,C,D).

\section{Irisin enters cells and plays a role through integrin aVB5 receptors}

After 8-weeks of nicotine exposure and 4-weeks of simultaneous irisin and cilengitide exposure, the Nicotine + Irisin + Cilengitide group was observed to reverse the effect of irisin on nicotine-induced medium thickening (mean 56.41 vs. $48.49 \mu \mathrm{m} ; \mathrm{P}=0.0201$, Figure $1 A, B$ ), macrophage infiltration (CD68 positive area $2.994 \%$ vs. $1.633 \% ; \mathrm{P}=0.0112$, Figure $1 A, C)$, and smooth muscle cell proliferation ( $\alpha$-SMA positive area $6.148 \%$ vs. $4.182 \%$; 
A
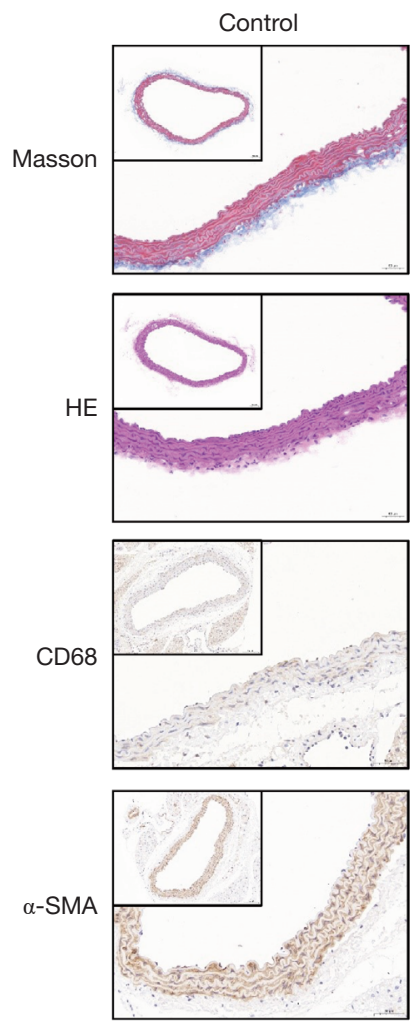

B

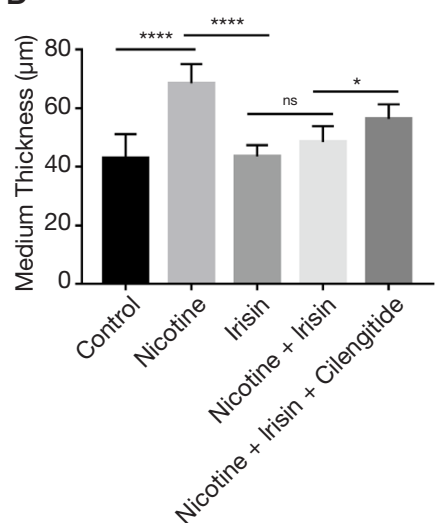

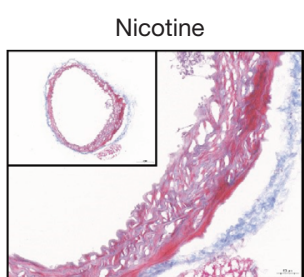
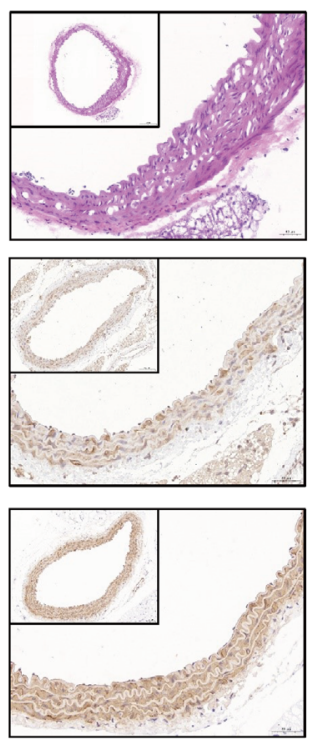

C

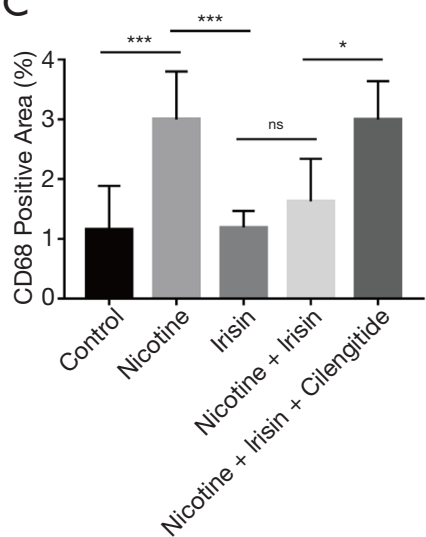

Irisin
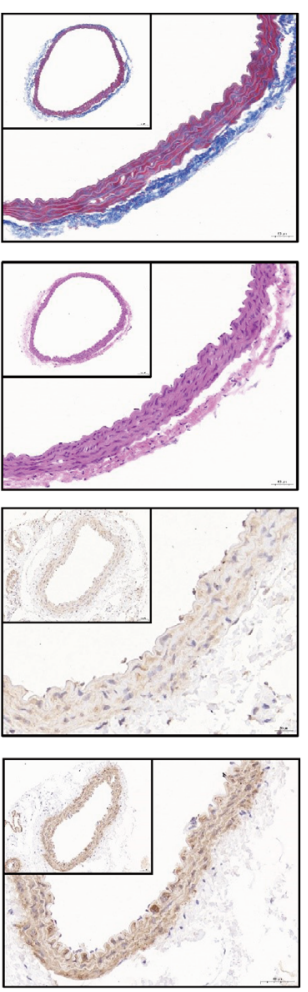

$\mathrm{D}$

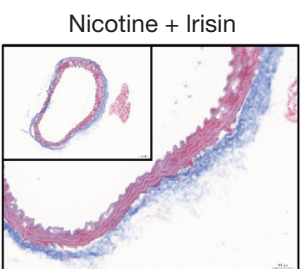

Nicotine + Irisin + Cilengitide
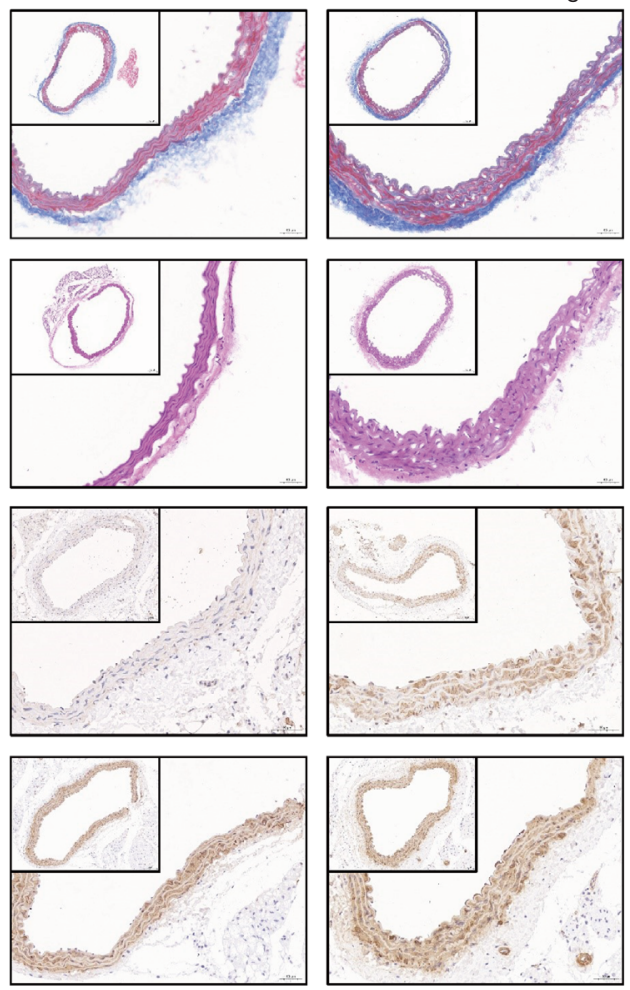

E

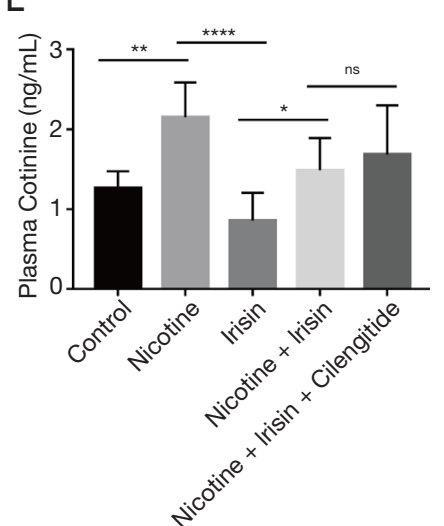

Figure 1 Nicotine increases aortic atherosclerosis lesion size in Apoe-/- mice. (A) Lesion area in murine aortic roots from the Control, Nicotine, Irisin, Nicotine + Irisin, and Nicotine + Irisin + Cilengitide groups were determined by Masson staining, HE staining, and immunohistochemical staining for CD68 and $\alpha$-SMA. (B) Medium thickness was measured based on Masson staining and was presented as mean thickness $\pm \mathrm{SD}$. (C) CD68 is a marker for macrophages. Quantification of positive CD68 staining area indicates macrophage infiltration. (D) $\alpha$-SMA is a marker for smooth muscle cells. Quantification of positive $\alpha$-SMA staining area indicates smooth muscle cell proliferation. (E) The quantification of plasma cotinine level was used to demonstrate the establishment of the nicotine intervention animal model. ${ }^{*} \mathrm{P}<0.05,{ }^{* *} \mathrm{P}<0.01,{ }^{* *} \mathrm{P}<0.001$, and ${ }^{* * * *} \mathrm{P}<0.0001$ by one-way ANOVA and student's $t$-test. Data are mean $\pm \mathrm{SD}$; ns, no significance, scale bar $=50 \mu \mathrm{m}$ in amplified view, $100 \mu \mathrm{m}$ in whole section view, $\mathrm{n}=8$. 
$\mathrm{P}=0.0323$, Figure $1 A, D)$ in the aortic root and ascending aorta, compared with the Nicotine + Irisin group. However, no significant difference in macrophage infiltration (CD68 positive area $2.994 \%$ vs. $2.997 \% ; \mathrm{P}>0.05$, Figure $1 A, C)$ and smooth muscle cell proliferation ( $\alpha$-SMA positive area $6.148 \%$ vs. $6.045 \% ; \mathrm{P}>0.05$, Figure $1 A, D)$ was observed between the Nicotine + Irisin + Cilengitide and Nicotine groups. Yet, the Nicotine + Irisin + Cilengitide group was still observed to inhibit nicotine-induced medium thickening (mean 56.41 vs. $68.51 \mu \mathrm{m} ; \mathrm{P}=0.0124$, Figure $1 A, B)$, suggesting that irisin has multiple pathways into the cell, such as endocytosis, to reverse nicotine-induced medium thickening. The administration of integrin $\alpha \mathrm{V} \beta 5$ inhibitor cilengitide was found to antagonize the effect of irisin in ameliorating macrophage infiltration and smooth muscle cell proliferation.

\section{Irisin reverses nicotine-mediated PI3K pathway activation in atherosclerotic aortic tissue}

According to previous human umbilical vein endothelial cells (HUVECs) RNA-sequencing results (data not shown), we found that the irisin-related integrin-PI3K pathway was activated after the administration of nicotine, while irisin could reverse nicotine-induced PI3K activation. In order to explore the molecular mechanism of irisin on nicotine-mediated medium thickening, we used the aortas of the five groups of Apoe-/- mice to study the expression of PI3K, AKT, p21, and p27. Western blot results showed that the band intensities of PI3K, AKT, p-AKT (T308), p-AKT (S473), and P21 were significantly stronger in the Nicotine group compared to the Control group (Figure $2 A, B, C, D, E, F$ ), while $\mathrm{P} 27$ was significantly weaker in the Nicotine group compared to the Control group (Figure $2 A, G$ ). Moreover, the expression level of PI3K in the Nicotine + Irisin group was lower than that of Nicotine group, suggesting that irisin could inhibit nicotine-mediated PI3K activation. Compared with the Nicotine group, the expression levels of AKT, p-AKT (T308), and p-AKT (S473) in the Nicotine + Irisin group were up-regulated, suggesting that irisin activated AKT. The expression of P21 in the Irisin group was up-regulated compared to the Nicotine group, and there was no significant difference in P21 between the Nicotine + Irisin and Nicotine groups, suggesting that irisin intervention alone could promote the expression of $\mathrm{P} 21$ and nicotine could antagonize it. The level of P27 in the Nicotine + Irisin group was higher than that in the Nicotine group, but there was no significant difference between the Irisin and Control groups, which is consistent with the results of PI3K, suggesting that irisin could reverse nicotine-mediated P27 down-regulation.

The protein expression of AKT was consistent with the RT-PCR results (Figure $3 A$ ). Compared with the Nicotine group, the expression of CDKN1A (P21, Figure 3B) in the Nicotine + Irisin group was up-regulated, while the protein level of P21 in the Nicotine + Irisin group was not significantly different. Also, there was no significant difference in CDKN1B (P27, Figure 3C) between the Nicotine and Control groups; however, compared with the Nicotine + Irisin group, irisin decreased the expression level of CDKN1B. It is worth noting that compared with the Nicotine group, the Nicotine + Irisin group exhibited further activated expression of phosphatase and tensin homolog (PTEN) (Figure 3D), suggesting that Irisin may activate PTEN to inhibit PI3K function.

\section{Irisin inbibits aortic atherosclerosis through integrin $\alpha V \beta 5$ receptors}

As a specific inhibitor of the integrin $\alpha \mathrm{V} \beta 5$ receptor, cilengitide was used to explore whether irisin enters the cell through integrin $\alpha \mathrm{V} \beta 5$ receptors and thus plays a further role. Western blot results showed that the protein levels of PI3K in the Nicotine + Irisin + Cilengitide group were reversed compared to the Nicotine + Irisin group (Figure $4 A, B)$. The Administration of cilengitide has no significant effect on the expression levels of AKT, compared with the Nicotine + Irisin group (Figure 4C). The expression of p-AKT (T308) was extremely prohibited after the administration of cilengitide, while it had no significant influence on the expression of p-AKT (S473) (Figure 4D,E). The expression levels of P21 in Nicotine + Irisin + Cilengitide group exhibited significantly increased compared to the Nicotine + Irisin group (Figure 4F), which was contrary to the effect of cilengitide on P27 (Figure $4 G)$. This further confirmed that irisin enters cells through integrin $\alpha \mathrm{V} \beta 5$ receptor and activates the expression of $\mathrm{P} 27$ by inhibiting the expression of PI3K.

However, different from the results of the Western Blot, the RT-PCR results indicated that the administration of cilengitide reversed nicotine-induced AKT3 up-regulation (Figure $3 A$ ), as the nicotine-induced AKT3 up-regulation was consistent with the results of the Western Blot. The mRNA levels of both CDKN1A and CDKN1B in the Nicotine + Irisin + Cilengitide group were also reversed compared to the Nicotine + Irisin group (Figure 3B,C). The 
A
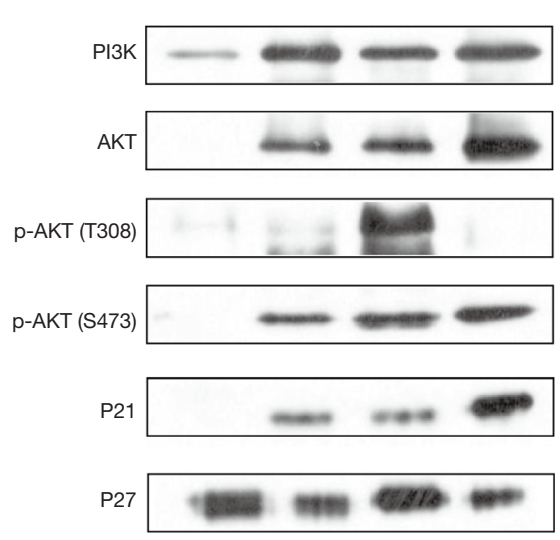

$\beta$-actin

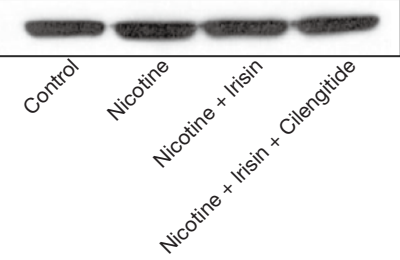

B
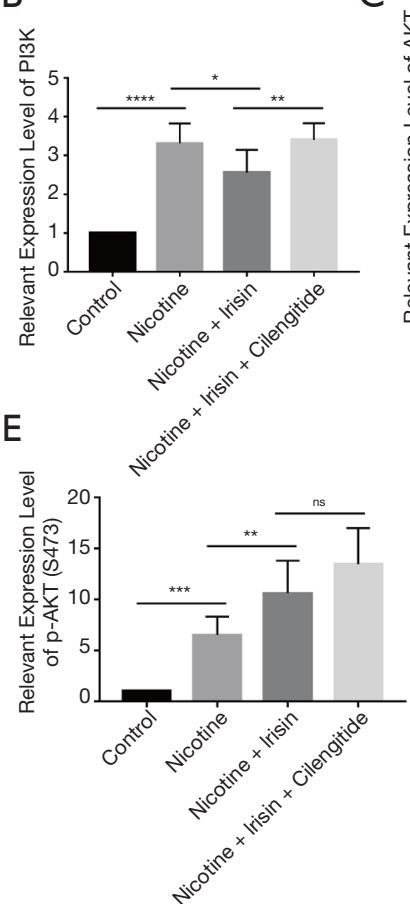

C

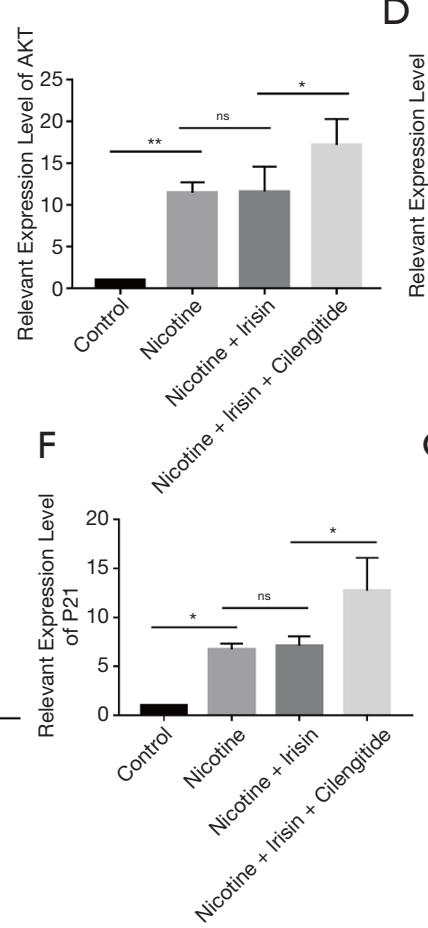

D

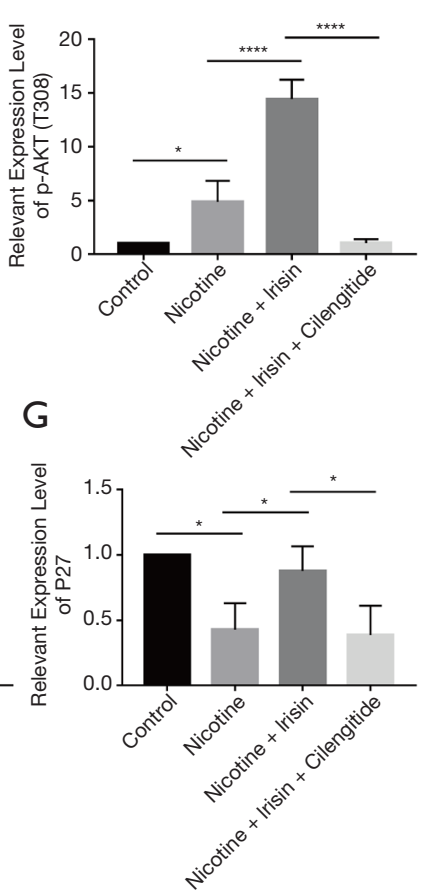

Figure 2 Irisin reverses the nicotine-mediated PI3K pathway activation in aortic atherosclerotic lesions of Apoe-/- mice. (A) Immunoblotting bands of PI3K, AKT, p-AKT (T308), p-AKT (S473), P21, and P27 from the Control, Nicotine, Irisin, and Nicotine + Irisin groups were presented. $\beta$-actin was used as housekeeping control. (B,C,D,E,F,G) Quantification of PI3K, AKT, p-AKT (T308), p-AKT (S473), P21, and P27 protein levels among the four groups. All results were normalized to the expression level of $\beta$-actin and presented as the fold change of the Control group. ${ }^{*} \mathrm{P}<0.05,{ }^{* *} \mathrm{P}<0.01,{ }^{* * *} \mathrm{P}<0.001$, and ${ }^{* * * *} \mathrm{P}<0.0001$ by one-way ANOVA. Data are mean $\pm \mathrm{SD} ; \mathrm{ns}$, no significance, $\mathrm{n}=8$.

A

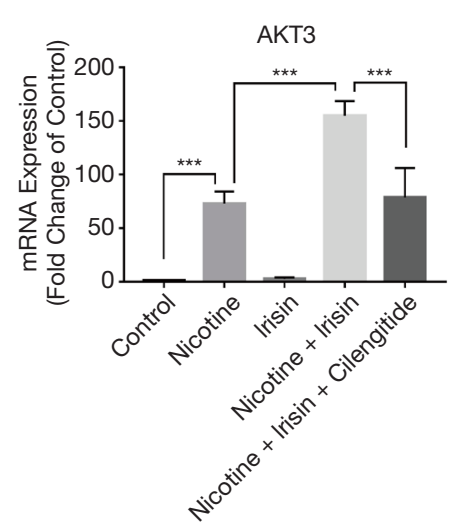

B

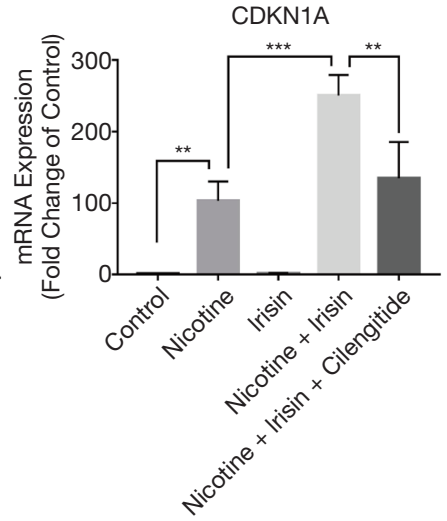

C

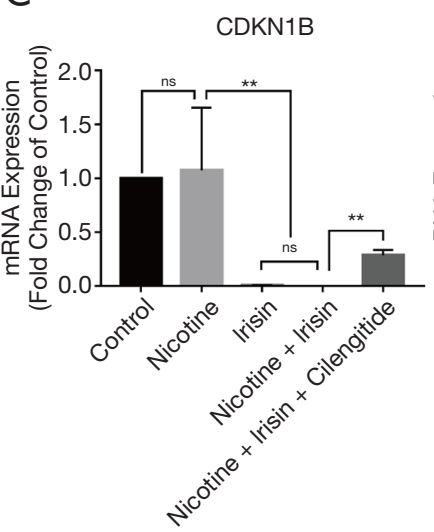

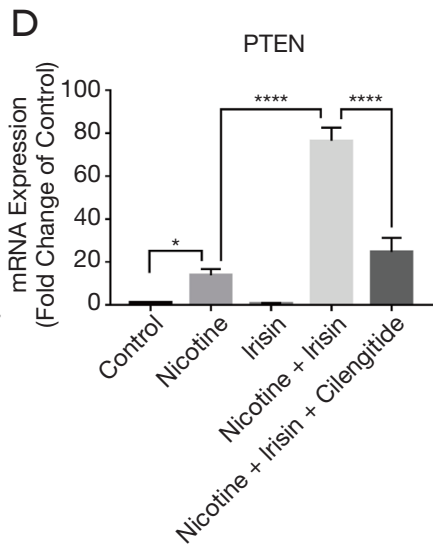

Figure 3 Effect of nicotine, irisin, and cilengitide on the expression levels of AKT3, CDKN1A, CDKN1B, and PTEN in the aortas of Apoe/- mice. (A-D) The mRNA levels of AKT3, CDKN1A (P21), CDKN1B (P27), and PTEN in the Control, Nicotine, Irisin, Nicotine + Irisin, and Nicotine + Irisin + Cilengitide groups were determined by quantitative RT-PCR. All results were normalized to the expression level of ACTB and presented as the fold change of the Control group. ${ }^{*} \mathrm{P}<0.05,{ }^{* *} \mathrm{P}<0.01,{ }^{* * *} \mathrm{P}<0.001$, and ${ }^{* * * *} \mathrm{P}<0.0001$ by one-way ANOVA and student's $t$-test. Data are mean $\pm \mathrm{SD}$; ns, no significance, $\mathrm{n}=8$. 

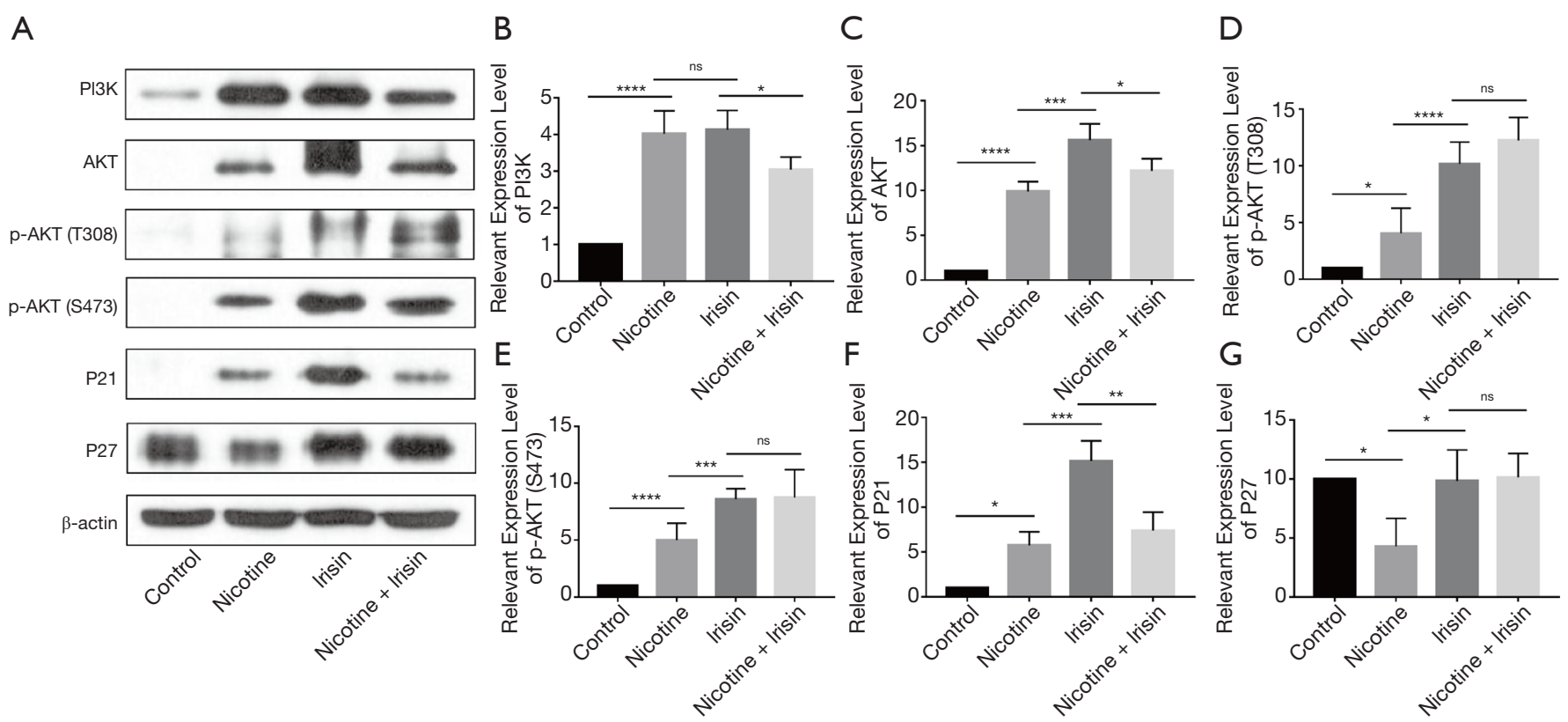

Figure 4 Irisin inhibits the nicotine-mediated PI3K pathway activation in aortic atherosclerotic lesions of Apoe-/- mice via integrin $\alpha \mathrm{V} \beta 5$. (A) Immunoblotting bands of PI3K, AKT, p-AKT (T308), p-AKT (S473), P21, and P27 from the Control, Nicotine, Nicotine + Irisin, and Nicotine + Irisin + Cilengitide groups were presented. $\beta$-actin was used as housekeeping control. (B,C,D,E,F,G) Quantification of PI3K, AKT, p-AKT (T308), p-AKT (S473), P21, and P27 protein level among the four groups. All results were normalized to the expression level of $\beta$-actin and presented as the fold change of the Control group. ${ }^{*} \mathrm{P}<0.05,{ }^{* *} \mathrm{P}<0.01,{ }^{* * *} \mathrm{P}<0.001$, and ${ }^{* * * *} \mathrm{P}<0.0001$ by one-way $\mathrm{ANOVA}$. Data are mean $\pm \mathrm{SD}$; ns, no significance, $\mathrm{n}=8$.

administration of cilengitide reversed the level of PTEN (Figure 3D), suggesting that irisin may activate PTEN through the integrin $\alpha \mathrm{V} \beta 5$ receptor, thus inhibiting PI3K and promoting the up-regulation of $\mathrm{P} 27$ to inhibit vascular smooth muscle proliferation and macrophage infiltration. Intima-media thickening and foam cell formation are thereby inhibited in order to further inhibit atherosclerosis.

\section{Discussion}

Irisin is a recently discovered myokine, and is considered to be a promising drug for cardiovascular disease treatment by improving vascular function. However, little is known about the role of irisin in the progression of atherosclerosis. Previous studies have demonstrated that irisin promotes the proliferation of HUVECs through the ERK signaling pathway and protects HUVECs from apoptosis by regulating the expression of Bcl-2, Bax, and Caspase $(18,19)$, thereby maintaining the dynamic balance of endothelial cells and promoting angiogenesis $(15,20)$. It has been further confirmed that irisin could significantly reduce the aortic atherosclerotic lesion area in Apoe-/- mice fed on a high-cholesterol diet (19) and suppressed the neointimal hyperplasia of the carotid artery on a carotid partial ligation model of Apoe-/- mice fed with a high-fat diet by upregulating the expression of microRNA126-5p through the ERK signaling pathway (20). However, there is no study elucidating the protective mechanism of irisin in smoking/ nicotine-related atherosclerosis.

In this study, nicotine was confirmed to promote arterial medium thickening, macrophage infiltration, and smooth muscle cell proliferation, which can be reversed by irisin in Apoe-/- mice. At the same time, the administration of cilengitide, an integrin $\alpha \mathrm{V} \beta 5$ inhibitor, to abolish a certain part of the irisin effect confirmed that irisin plays a role in arterial walls through integrin $\alpha \mathrm{V} \beta 5$, which provides further evidence for the molecular mechanism of irisin as a treatment candidate for atherosclerosis. Furthermore, this study confirmed that irisin inhibits the activation of nicotine-mediated PI3K and promotes the expression of cyclin-dependent protein kinase inhibitors such as P27, thus inhibiting medium thickening and atherosclerotic 
progression. Meanwhile, the expression level of p-AKT (T308) was down-regulated by cilengitide, while the expression level of p-AKT (S473) was not affected by the administration of cilengitide. Considering that the phosphorylation of AKT (S473) is associated with the full activation of AKT function, the expression level of p-AKT (S473) is similar to total AKT. The changing trend of protein and mRNA levels of P27 was consistent with the changing trend of PI3K expression, but not in accordance with the trend of AKT activation, suggesting that irisin may inhibit nicotine-mediated medium thickening and atherosclerosis via the integrin $\alpha \mathrm{V} \beta 5 / \mathrm{PI} 3 \mathrm{~K} / \mathrm{P} 27$ pathway, bypassing AKT.

As an inhibitor of PI3K, PTEN was included in this study to explain how irisin inhibits the activation of PI3K. An increased mRNA level of PTEN was observed in the Nicotine + Irisin group compared with Nicotine group, and the administration of cilengitide down-regulated the expression of PTEN, suggesting that irisin may inhibit smooth muscle cell proliferation, macrophage infiltration, and atherosclerosis progression through the integrin $\alpha \mathrm{V} \beta 5$ / PTEN/PI3K/P27 pathway.

The key limitation of this study is that the explored downstream only included cell cycle- and proliferationrelated pathways such as $\mathrm{PI} 3 \mathrm{~K} / \mathrm{AKT} / \mathrm{P} 21 / \mathrm{P} 27$, which can only explain the mechanism of irisin reversing nicotinemediated smooth muscle cell proliferation, and does not further elaborate on the molecular mechanism of irisin against nicotine-mediated macrophage infiltration.

In conclusion, we confirmed that irisin ameliorates nicotine-mediated medium thickening, smooth muscle cell proliferation, macrophage infiltration, and atherosclerosis progression via the integrin $\alpha \mathrm{V} \beta 5$ receptor to inhibit PI3K and activate P27 in Apoe-/- mice. PTEN is a potential target for the inhibitory effect of irisin on PI3K. These findings provide further evidence of the mechanism of irisin for the treatment of atherosclerosis.

\section{Acknowledgments}

The authors would like to acknowledge Dr. Wenjun Guo for her guidance and assistance in this research.

Funding: This study received funding from the National Natural Science Foundation of China (No. 82070498), the Natural Science Foundation of Beijing Municipality (No. 7182131), the Fundamental Research Funds for the Central Universities (No. 3332019129), and the Non-profit Central Research Institute Fund of Chinese Academy of Medical
Science (No. 2019XK320004).

\section{Footnote}

Reporting Checklist: The authors have completed the ARRIVE reporting checklist. Available at http://dx.doi. org/10.21037/atm-21-2072

Data Sharing Statement: Available at http://dx.doi. org/10.21037/atm-21-2072

Conflicts of Interest: All authors have completed the ICMJE uniform disclosure form (available at http://dx.doi. org/10.21037/atm-21-2072). The authors report that this study received funding from the National Natural Science Foundation of China (No. 82070498), the Natural Science Foundation of Beijing Municipality (No. 7182131), the Fundamental Research Funds for the Central Universities (No. 3332019129), and the Non-profit Central Research Institute Fund of Chinese Academy of Medical Science (No. 2019XK320004). The authors have no other conflicts of interest to declare.

Ethical Statement: The authors are accountable for all aspects of the work in ensuring that questions related to the accuracy or integrity of any part of the work are appropriately investigated and resolved. All animal experiments were conducted according to the guidelines of the National Health and Family Planning Commission of the People's Republic of China and approved by the Animal Ethics Committee of Peking Union Medical College Hospital, Chinese Academy of Medical Sciences (approval number JS-2335).

Open Access Statement: This is an Open Access article distributed in accordance with the Creative Commons Attribution-NonCommercial-NoDerivs 4.0 International License (CC BY-NC-ND 4.0), which permits the noncommercial replication and distribution of the article with the strict proviso that no changes or edits are made and the original work is properly cited (including links to both the formal publication through the relevant DOI and the license). See: https://creativecommons.org/licenses/by-nc-nd/4.0/.

\section{References}

1. Ungvari Z, Tarantini S, Sorond F, et al. Mechanisms of Vascular Aging, A Geroscience Perspective: JACC Focus 


\section{Page 10 of 10}

Seminar. J Am Coll Cardiol 2020;75:931-41.

2. Roth GA, Johnson C, Abajobir A, et al. Global, Regional, and National Burden of Cardiovascular Diseases for 10 Causes, 1990 to 2015. J Am Coll Cardiol 2017;70:1-25.

3. Wang C, Chen $\mathrm{H}$, Zhu W, et al. Nicotine Accelerates Atherosclerosis in Apolipoprotein E-Deficient Mice by Activating $\alpha 7$ Nicotinic Acetylcholine Receptor on Mast Cells. Arteriosclerosis, thrombosis, and vascular biology 2017;37:53-65.

4. Barua RS, Ambrose JA, Srivastava S, et al. Reactive oxygen species are involved in smoking-induced dysfunction of nitric oxide biosynthesis and upregulation of endothelial nitric oxide synthase: an in vitro demonstration in human coronary artery endothelial cells. Circulation 2003;107:2342-7.

5. Yoshiyama S, Chen Z, Okagaki T, et al. Nicotine exposure alters human vascular smooth muscle cell phenotype from a contractile to a synthetic type. Atherosclerosis 2014;237:464-70.

6. Boström P, Wu J, Jedrychowski MP, et al. A PGC1- $\alpha$ dependent myokine that drives brown-fat-like development of white fat and thermogenesis. Nature 2012;481:463-8.

7. Schumacher MA, Chinnam N, Ohashi T, et al. The structure of irisin reveals a novel intersubunit betasheet fibronectin type III (FNIII) dimer: implications for receptor activation. J Biol Chem 2013;288:33738-44.

8. Mazur-Bialy AI, Pochec E, Zarawski M. AntiInflammatory Properties of Irisin, Mediator of Physical Activity, Are Connected with TLR4/MyD88 Signaling Pathway Activation. Int J Mol Sci 2017;18:701.

9. Kim H, Wrann CD, Jedrychowski M, et al. Irisin Mediates Effects on Bone and Fat via alphaV Integrin Receptors. Cell 2019;178:507-8.

10. Fu J, Han Y, Wang J, et al. Irisin Lowers Blood Pressure by Improvement of Endothelial Dysfunction via AMPK-AkteNOS-NO Pathway in the Spontaneously Hypertensive Rat. J Am Heart Assoc 2016;5:e003433.

11. Li H, Shen J, Wu T, et al. Irisin Is Controlled by Farnesoid

\section{Li et al. Irisin ameliorates nicotine-mediated atherosclerosis}

$\mathrm{X}$ Receptor and Regulates Cholesterol Homeostasis. Front Pharmacol 2019;10:548.

12. Byun K, Lee S. The Potential Role of Irisin in Vascular Function and Atherosclerosis: A Review. Int J Mol Sci 2020;21:7184.

13. Zhang $M, X u Y$, Jiang L. Irisin attenuates oxidized lowdensity lipoprotein impaired angiogenesis through AKT/mTOR/S6K1/Nrf2 pathway. J Cell Physiol 2019;234:18951-62.

14. Zhang Y, Li R, Meng Y, et al. Irisin stimulates browning of white adipocytes through mitogen-activated protein kinase p38 MAP kinase and ERK MAP kinase signaling. Diabetes 2014;63:514-25.

15. Rabiee F, Lachinani L, Ghaedi S, et al. New insights into the cellular activities of Fndc5/Irisin and its signaling pathways. Cell Biosci 2020;10:51.

16. Xiong XQ, Geng Z, Zhou B, et al. FNDC5 attenuates adipose tissue inflammation and insulin resistance via AMPK-mediated macrophage polarization in obesity. Metabolism 2018;83:31-41.

17. Xiong XQ, Chen D, Sun HJ, et al. FNDC5 overexpression and irisin ameliorate glucose/lipid metabolic derangements and enhance lipolysis in obesity. Biochim Biophys Acta 2015;1852:1867-75.

18. Song H, Wu F, Zhang Y, et al. Irisin promotes human umbilical vein endothelial cell proliferation through the ERK signaling pathway and partly suppresses high glucose-induced apoptosis. PLoS One 2014;9:e110273.

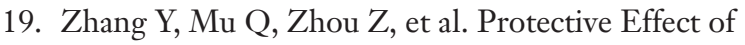
Irisin on Atherosclerosis via Suppressing Oxidized Low Density Lipoprotein Induced Vascular Inflammation and Endothelial Dysfunction. PLoS One 2016;11:e0158038.

20. Zhang $\mathrm{Y}$, Song $\mathrm{H}$, Zhang $\mathrm{Y}$, et al. Irisin Inhibits Atherosclerosis by Promoting Endothelial Proliferation Through microRNA126-5p. J Am Heart Assoc 2016;5:e04031.

(English Language Editor: A. Kassem)
Cite this article as: Li K, Chen J, Wang C, Shao J, Lai Z, Yu X, Du F, Gao R, Wang J, Liu B. Irisin ameliorates nicotinemediated atherosclerosis via inhibition of the PI3K pathway. Ann Transl Med 2021;9(9):805. doi: 10.21037/atm-21-2072 